\title{
The Benefit of Postoperative Radiotherapy and Extending Neck Dissection in pT1-2 Oral Squamous Cell Carcinoma With a Single Ipsilateral Cervical Lymph Node Metastasis (pN1)
}

\author{
UBAI ALSHARIF ${ }^{1,2,3}$, DANIEL STELLER ${ }^{1}$, MOHAMED FALOUGY $^{1}$, \\ LARS THARUN $^{4}$, DIRK RADES ${ }^{5}$ and SAMER G. HAKIM ${ }^{1}$ \\ ${ }^{1}$ Department of Oral and Maxillofacial Surgery, \\ University Hospital Schleswig-Holstein (Campus Lübeck), Lübeck, Germany; \\ ${ }^{2}$ Department of Oral and Maxillofacial Surgery, Dortmund General Hospital, Dortmund, Germany; \\ ${ }^{3}$ School of Dentistry, Faculty of Health, Witten/Herdecke University, Witten, Germany; \\ ${ }^{4}$ Institute of Pathology, University Hospital Schleswig-Holstein (Campus Lübeck), Lübeck, Germany; \\ ${ }^{5}$ Department of Radiation Oncology, University Hospital Schleswig-Holstein (Campus Lübeck), Lübeck, Germany
}

\begin{abstract}
Background/Aim: We compared postoperative radiotherapy (PORT) to surgery only (SO), and supraomohyoidal neck dissection (SOHND) to modified radical neck dissection (MRND) in patients with pT1-T2 squamous cell carcinomas of the oral cavity (OSCC) and a single cervical lymph node metastasis ( $p N 1)$ in terms of overall survival (OS), oral cancer specific survival (OCSS), and regional recurrencefree survival (RRFS), in a prospective cohort study. Patients and Methods: We included patients with pTI-T2 pN1 OSCC with no distant metastasis and estimated the survival probabilities using the Kaplan-Meier method and calculated hazards ratios (HR) for PORT vs. SO and MRND vs. SOHND using adjusted Cox regression models. Results: A total of 51 patients (26 SO vs. 25 PORT, 9 SOHND vs. 42 MRND) were evaluated. Patients who received PORT were more likely to be younger and healthier. OS at 5 years was $41 \%$ and $87 \%$ in the SO and PORT groups, respectively. OS at 5 years was 52\% and $67 \%$ in the in the SOHND and MRND groups, respectively. Both OCSS and RRFS were improved by PORT.
\end{abstract}

This article is freely accessible online.

Correspondence to: Samer G. Hakim, MD, DMD, Ph.D., Department of Oral and Maxillofacial Surgery, University Hospital Schleswig-Holstein (Campus Lübeck), Lübeck, D-23538, Germany. Tel: +49 45150042501, e-mail: samer.hakim@uksh.de

Key Words: Squamous cell carcinoma, oral cancer, pN1, survival, adjuvant radiotherapy, metastatic lymphadenopathy, neck dissection, postoperative radiotherapy.
Extending neck dissection was not associated with improved OS $(H R=0.83)$. Conclusion: PORT is associated with preferable OS, OCSS, and RRFS in pT1-2 pN1 oral cancer and should be recommended regularly.

Postoperative radiotherapy (PORT) in oral squamous cell carcinoma (OSCC) is usually indicated in cases where the primary tumor exceeds T2 size, is incompletely removed or carries an inherent risk of recurrence (e.g., deep infiltrative growth and/or with perineural or lymphovascular invasion). Further, PORT is recommended in cases where locoregional spread into cervical lymph nodes is evident or anticipated. However, the advantage of PORT in intermediate risk OSCC such as cases of small (pT1-2) tumors with a single lymph node metastasis ( $\mathrm{pN} 1)$ without extracapsular spread is scarce and the recommendations of PORT have been inconsistent (1).

The Clinical Practice Guideline of the American Society of Clinical Oncology (ASCO) published in 2019 does not recommend PORT in $\mathrm{pN} 1$ cases with no further histologic risk factors (2). The recommendation implies a sufficient quality of the neck dissection based on the number of lymph nodes encountered and sets a cutoff of at least 18 lymph nodes. In cases where lymph node yield is lower than 18 , adjuvant radiotherapy is recommended.

In contrast, the current German guideline for the management of oral cancer states that adjuvant radiotherapy may be offered for patients with pT1-2 pN1 tumors without extranodal extension (ENE-) $(3,4)$. Nodal yield was not considered a quality criterion for a reliable neck dissection.

Apart of the many controversial recommendations regarding this question, there are several factors that complicate the 
evaluation of the impact of PORT on locoregional control and survival in patients with a $\mathrm{pN} 1$ constellation. First, the prevalence of the $\mathrm{pN} 1$ situation in small OSCC is low to be encountered within prospective randomized trials. Second, the quality of non-randomized studies addressing this question is compromised by methodological shortcomings. For example, PORT is generally recommended in patients with proper overall health condition, meaning that older patients in a limited general condition mostly do not receive PORT. This is especially relevant when considering the overall survival (OS) as the primary outcome in those studies. Furthermore, oral cancer death or regional recurrence have not yet been investigated considering competing survival events (e.g., death from causes other than oral cancer).

To address the role of PORT in the $\mathrm{pN} 1$ situation of pT1-T2 OSCC, we compared different survival outcomes in patients who received PORT with those who were treated surgically only (SO) in a prospectively maintained cohort. We further accounted for the extent of neck dissection and for competing survival events.

\section{Patients and Methods}

Study population. All patients with a primary OSCC without distant metastasis from a cohort of 1,089 patients were identified within the tumor data bank of the Department of Maxillofacial Surgery of the University Medical Centre of Lübeck, Germany. All patients underwent primary diagnostic investigation and therapy between 1992 and 2019. For this study, we only included curatively treated patients who had a pT1 or pT2 OSCC and a single concomitant ipsilateral cervical lymph node metastasis (pN1) without extra-nodal spread (ENE-). Patients with a distant metastasis, cancer of unknown primary, history of head and neck cancer with or without irradiation were excluded. Figure 1 shows the criteria for exclusion.

Patients' data were obtained from a prospectively maintained single-center cohort and included patients' demographics, risk factors and clinical tumor characteristics. Treatment decisions were available at the baseline and at each follow-up. We used the updated version of the Charlson's comorbidity index (CCI) to capture comorbidities and categorized CCI into no significant comorbidities (score $=0$ ), and at least one score point (CCI score $\geq 1$ ) (5).

Although the recommendations for surgery and PORT were made by the local Head and Neck Cancer multidisciplinary team, not all patients adhered to this recommendation. Reasons for non-adherence were prolonged wound healing period exceeding 6 weeks, serious associated comorbidities or refusing the PORT as such.

Follow-up and survival endpoints. All patients underwent routine recall and related clinical investigations every three months in the first two years and every six months for the last three years of the 5 years follow-up period. This follow-up included clinical, sonographic and radiologic assessment and ended when patients either fulfilled 5 years of complete disease-free follow-up, decided to drop out from the regular aftercare, died or the follow-up endpoint on the 31 of March 2019 was reached. Patients who were alive at the end of follow-up were censored. All survival durations

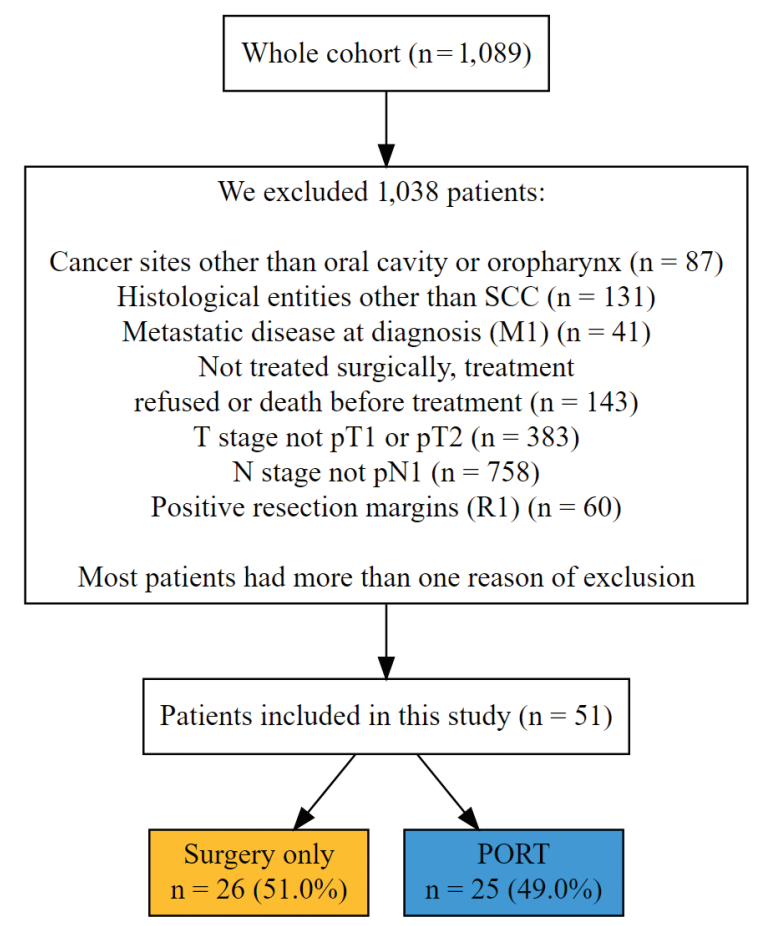

Figure 1. Flow-chart of patients' selection and number of included patients by group.

were measured from the time point of the initial diagnosis. The endpoint of OS was death from any cause; the endpoint of oral cancer specific survival (OCSS) was death from oral cancer; the endpoint of locoregional recurrence-free survival (LRFS) was the local or neck recurrence, while regional recurrence-free survival (RRFS) was measured until a neck recurrence occurred.

Ethics. All participants signed consent forms allowing their data to be collected and used anonymously for academic research on admission. The study was approved by the ethics review committee of the University of Lübeck (Approval number: 16-272A).

Statistical analysis. The baseline data were tabulated after calculating medians and interquartile range (IQR) for skewed variables. The median, 2- and 5-years survival probabilities as well as $95 \%$ corresponding confidence intervals (CI) were estimated using the Kaplan-Meier method for survival analysis for OS and cumulative events for OCSS, RRFS and LRFS. We estimated adjusted hazards ratios (HR) and corresponding 95\% CI for PORT and associated risk factors using Cox's proportional hazards regression models. For OCSS, RRFS and LRFS, we estimated adjusted cause-specific HRs in competing risks scenarios, which means that all three outcomes had death from any cause other than oral cancer as a competing risk (6). When inspecting Schoenfeld's residual plots (7), the proportionality of hazards was deemed violated by CCI score and tumor size. Thus, we stratified the Cox's models by these two variables. All statistical analyses were performed using R Statistical Software (version 4.0.4; R Foundation for Statistical Computing, Vienna, Austria). 


\section{Results}

In our cohort, $10 \%$ of all completely resected non-metastatic pT1-2 SCC showed histologically confirmed singular lymph node metastasis. In total, 51 patients fulfilled the inclusion criteria of this study, 25 (49\%) patients received PORT, while $26(51 \%)$ had SO as the sole curative treatment. There were substantial differences between the groups at the baseline in terms of age, general health condition and tumor size. Patients in the PORT group were on average 13 years younger [Median age: 57 vs. 70] and healthier [no comorbidities: $23(92 \%)$ vs. $12(46 \%)$ ] than patients in the SO group. However, patients in the SO group were more likely to have T1 tumors [16 (62\%) vs. 4 (16\%)].

The distribution of other risk factors, such as smoking, alcohol consumption, cancer subsite and differentiation were balanced between groups. More than two thirds of patients in both groups had a positive history of smoking, and more than half of them had a history of excessive alcohol consumption; the anterior tongue was the most common site for the primary tumor in both groups, and the majority of tumors were moderately differentiated.

In the preoperative staging, cervical lymph node metastases were identified correctly as being a single lymph node metastasis in almost $40 \%$ of cases in both groups. All tumors were removed with sufficient resection margins. An extension of the neck dissection to levels IV and $\mathrm{V}$ was undertaken in similar proportions in both groups [22 (85\%) in the SO group vs. $20(80 \%)$ in the PORT group]. Twentyeight patients $(54.9 \%)$ required a microvascular free-flap reconstruction, while twenty-five patients $(45.1 \%)$ received primary defect closure. Patients' characteristics at the timepoint of the initial treatment are presented in Table I.

Survival. The follow-up ranged from 9 months to 9 years with a median OS time of 6.6 years among survivors. In a cumulative follow-up duration of 204 person-years, 24 patients died, resulting in a death rate of 11.7 deaths per 100 person-years.

After 5 years of follow-up, 17 (33\%) patients died, 10 (20\%) patients died due to oral cancer and $5(9.8 \%)$ died from other causes. The cause of death was unknown in two $2(3.9 \%)$ patients. For the whole cohort, the survival probabilities at 5 years were $41 \%(25 \%-68 \%)$ and $87 \%(74 \%-100 \%)$ for the SO and PORT groups, respectively.

Within the follow-up period, $8(16 \%)$ patients developed regional disease recurrences; All neck recurrences occurred within the first 2.5 years after the initial treatment. In the SO group, $7(27 \%)$ patients developed regional recurrences while only one patient $1(4.0 \%)$ suffered from a regional recurrence in the PORT group. The rates of disease recurrence events are reported in Table II. Detailed survival estimates and event probabilities are presented in Table III and Figure 2.
We examined the impact of PORT on different survival outcomes using four adjusted Cox proportional hazards models while addressing competing risks when appropriate. Both CCI score and tumor size violated the proportional hazard assumption, thus we stratified the models for those two factors. In this selected cohort, PORT but not the extent of the neck dissection was associated with improved overall and disease specific survival. The adjusted hazards ratios for PORT were 0.07 (95\% CI=0.01-0.69; $p=0.023$ ) for RRFS, 0.06 $(95 \% \mathrm{CI}=0.01-0.31 ; \mathrm{p}<0.001)$ for LRFS, $0.19(95 \% \mathrm{CI}=0.04-$ $0.82 ; p=0.026)$ for OCSS and $0.22(95 \% \mathrm{CI}=0.05-1.03$; $p=0.054$ ) for OS. Table IV shows the hazards ratio for each of the predictors for OS, OCSS, LRFS, and RRFS.

\section{Discussion}

In the current understanding of tumor cell biology and the mechanisms of locoregional metastatic spread, two main scenarios may be raised to justify treatment strategy. Either the migrating tumor cells from the primary tumor pass the lymphatic vessels to the draining regional lymph nodes and thus alternate the local neoplastic disease into a locoregional one, requiring more than excision of the primary and the draining lymph nodes (neck dissection); or the migrating tumor cells do not play any pathological role between "departure" and "destination" at least in the early stage of metastasis (N1 situation). The therapeutic consequence of the first scenario is a recommendation for radiotherapy, for the second one - upon exclusion of further risk factors - is "wait and see."

In an effort to provide a sufficient answer and an evidencebased recommendation for the relatively rare case of pT1-2 pN1 situation, several retrospective studies have been carried out (8-14). Among the relatively inhomogeneous studies, including different adverse pathological features, competing risk factors and patients' characteristics, publications analyzing the US National Cancer Database have drawn attention, mainly because of the large number of patients included in the analyses (15-17). The National Cancer Database includes only the timepoint of death and does not consider further follow-up events. Hence, these studies were only able to examine the OS as a survival outcome. However, findings from our study suggest that patients who did not receive PORT were older and were more likely to suffer from co-morbidities. Thus, this negative pre-selection might have biased survival analyses and therefore, limits the validity of OS as an outcome as it may in part - explain why patients in the "wait and see" group died earlier than other participants.

None of the previous studies evaluated the RRFS and LRFS separately. These features, however, play a major role in the early stage pT1-2 pN1 tumors, since local tumor recurrence without lymph node involvement is related to the histopathologic features of the primary tumor (such as tumor cell remnants implicating early local recurrence), whereas 
Table I. Characteristics of study population by postoperative radiotherapy and neck dissection.

\begin{tabular}{|c|c|c|c|c|c|c|c|}
\hline & \multirow{2}{*}{$\begin{array}{c}\text { Overall } \\
\mathrm{N}=51\end{array}$} & \multicolumn{3}{|c|}{ Treatment } & \multicolumn{3}{|c|}{ Neck dissection } \\
\hline & & $\begin{array}{l}\text { Surgery only } \\
\mathrm{N}=26(51 \%)\end{array}$ & $\begin{array}{c}\text { PORT } \\
\mathrm{N}=25(49 \%)\end{array}$ & $p$-Value & $\begin{array}{c}\text { SOHND } \\
\mathrm{N}=9(18 \%)\end{array}$ & $\begin{array}{c}\text { MRND } \\
\mathrm{N}=42(82 \%)\end{array}$ & $p$-Value \\
\hline Age & $62(54-72)$ & $70(61-79)$ & $57(50-64)$ & $<0.001$ & $67(62-70)$ & $62(52-72)$ & 0.2 \\
\hline Gender & & & & 0.4 & & & 0.7 \\
\hline Male & $36(71 \%)$ & $17(65 \%)$ & $19(76 \%)$ & & $7(78 \%)$ & $29(69 \%)$ & \\
\hline Female & $15(29 \%)$ & $9(35 \%)$ & $6(24 \%)$ & & $2(22 \%)$ & $13(31 \%)$ & \\
\hline CCI score & & & & $<0.001$ & & & 0.7 \\
\hline 0 & $35(69 \%)$ & $12(46 \%)$ & $23(92 \%)$ & & $7(78 \%)$ & $28(67 \%)$ & \\
\hline 1 or more & $16(31 \%)$ & $14(54 \%)$ & $2(8.0 \%)$ & & $2(22 \%)$ & $14(33 \%)$ & \\
\hline Smoking & & & & 0.7 & & & 0.14 \\
\hline Never & $9(18 \%)$ & $4(15 \%)$ & $5(21 \%)$ & & $3(38 \%)$ & $6(14 \%)$ & \\
\hline Former or current & $41(82 \%)$ & $22(85 \%)$ & $19(79 \%)$ & & $5(62 \%)$ & $36(86 \%)$ & \\
\hline Missing & 1 & 0 & 1 & & 1 & 0 & \\
\hline Pack-Years & $28(1-44)$ & $30(4-50)$ & $22(3-39)$ & 0.4 & $0(0-21)$ & $30(13-48)$ & 0.077 \\
\hline Missing & 17 & 10 & 7 & & 4 & 13 & \\
\hline Alcohol consumption & & & & 0.8 & & & 0.2 \\
\hline None or moderate & $20(40 \%)$ & $10(38 \%)$ & $10(42 \%)$ & & $5(62 \%)$ & $15(36 \%)$ & \\
\hline Excessive & $30(60 \%)$ & $16(62 \%)$ & $14(58 \%)$ & & $3(38 \%)$ & $27(64 \%)$ & \\
\hline Missing & 1 & 0 & 1 & & 1 & 0 & \\
\hline Subsite & & & & 0.15 & & & 0.091 \\
\hline Anterior tongue & $22(43 \%)$ & $15(58 \%)$ & $7(28 \%)$ & & $3(33 \%)$ & $19(45 \%)$ & \\
\hline Gum & $8(16 \%)$ & $5(19 \%)$ & $3(12 \%)$ & & $3(33 \%)$ & $5(12 \%)$ & \\
\hline Floor of mouth & $9(18 \%)$ & $3(12 \%)$ & $6(24 \%)$ & & $0(0 \%)$ & $9(21 \%)$ & \\
\hline Palate & $2(3.9 \%)$ & $1(3.8 \%)$ & $1(4.0 \%)$ & & $1(11 \%)$ & $1(2.4 \%)$ & \\
\hline Cheek, Vestibule, retromolar & $5(9.8 \%)$ & $1(3.8 \%)$ & $4(16 \%)$ & & $2(22 \%)$ & $3(7.1 \%)$ & \\
\hline Oropharynx & $5(9.8 \%)$ & $1(3.8 \%)$ & $4(16 \%)$ & & $0(0 \%)$ & $5(12 \%)$ & \\
\hline Pathological tumor size (pT) & & & & $<0.001$ & & & $>0.9$ \\
\hline $\mathrm{T} 1$ & $20(39 \%)$ & $16(62 \%)$ & $4(16 \%)$ & & $3(33 \%)$ & $17(40 \%)$ & \\
\hline $\mathrm{T} 2$ & $31(61 \%)$ & $10(38 \%)$ & $21(84 \%)$ & & $6(67 \%)$ & $25(60 \%)$ & \\
\hline Clinical nodal status (cN) & & & & 0.7 & & & 0.4 \\
\hline No & $14(27 \%)$ & $8(31 \%)$ & $6(24 \%)$ & & $4(44 \%)$ & $10(24 \%)$ & \\
\hline N1 & $21(41 \%)$ & $10(38 \%)$ & $11(44 \%)$ & & $2(22 \%)$ & $19(45 \%)$ & \\
\hline $\mathrm{N} 2 \mathrm{a} / \mathrm{b}$ & $8(16 \%)$ & $3(12 \%)$ & $5(20 \%)$ & & $1(11 \%)$ & $7(17 \%)$ & \\
\hline $\mathrm{N} 2 \mathrm{c} / 3$ & $8(16 \%)$ & $5(19 \%)$ & $3(12 \%)$ & & $2(22 \%)$ & $6(14 \%)$ & \\
\hline Resection margins & & & & 0.039 & & & $>0.9$ \\
\hline R0 safe margins & $19(40 \%)$ & $13(54 \%)$ & $6(25 \%)$ & & $4(44 \%)$ & $15(38 \%)$ & \\
\hline R0 close margins & $29(60 \%)$ & $11(46 \%)$ & $18(75 \%)$ & & $5(56 \%)$ & $24(62 \%)$ & \\
\hline Missing & 3 & 2 & 1 & & 0 & 3 & \\
\hline Grade of differentiation & & & & 0.7 & & & 0.4 \\
\hline Well & $3(5.9 \%)$ & $2(7.7 \%)$ & $1(4.0 \%)$ & & $1(11 \%)$ & $2(4.8 \%)$ & \\
\hline Moderate & $32(63 \%)$ & $15(58 \%)$ & $17(68 \%)$ & & $4(44 \%)$ & $28(67 \%)$ & \\
\hline Poor & $16(31 \%)$ & $9(35 \%)$ & $7(28 \%)$ & & $4(44 \%)$ & $12(29 \%)$ & \\
\hline Free flap reconstruction & $28(55 \%)$ & $12(46 \%)$ & $16(64 \%)$ & 0.2 & $5(56 \%)$ & $23(55 \%)$ & $>0.9$ \\
\hline Chemotherapy & $7(14 \%)$ & $0(0 \%)$ & $7(28 \%)$ & 0.004 & $1(11 \%)$ & $6(14 \%)$ & $>0.9$ \\
\hline Type of neck dissection & & & & 0.7 & & & \\
\hline SOHND & $9(18 \%)$ & $4(15 \%)$ & $5(20 \%)$ & & & & \\
\hline MRND & $42(82 \%)$ & $22(85 \%)$ & $20(80 \%)$ & & & & \\
\hline
\end{tabular}

CCI: Updated Charlson's Comorbidity Index score; SOHND: supraomohyoidal neck dissection; MRND: modified radical neck dissection; PORT: postoperative radiotherapy.

only RRFS would reflect the inherent answer on the potential benefit of radiotherapy in $\mathrm{pN} 1$ cases.

A comparable large study by Shrime et al. analyzed the Surveillance, Epidemiology, and End Results database for survival in patients with pT1-2 pN1 OCSCC and could only assess a significant OS improvement in $\mathrm{pT} 2 \mathrm{pN} 1$ but not in pT1 pN1 tumors (18).

Besides the missing disease-free and disease-specific survival in the mentioned database studies, a crucial question for the recommendation of post-operative radiotherapy remains 
Table II. Event rates in 5 years stratified by treatment group.

\begin{tabular}{|c|c|c|c|c|c|}
\hline & \multirow{2}{*}{$\begin{array}{l}\text { Overall } \\
\mathrm{N}=51^{1}\end{array}$} & \multicolumn{2}{|c|}{ Treatment } & \multicolumn{2}{|c|}{ Neck dissection } \\
\hline & & $\begin{array}{c}\text { Surgery only } \\
\mathrm{N}=26(51 \%)^{1}\end{array}$ & $\begin{array}{c}\text { PORT } \\
\mathrm{N}=25(49 \%)^{1}\end{array}$ & $\begin{array}{c}\text { SOHND } \\
\mathrm{N}=9(18 \%)^{1}\end{array}$ & $\begin{array}{c}\text { MRND } \\
\mathrm{N}=42(82 \%)^{1}\end{array}$ \\
\hline \multicolumn{6}{|l|}{ Death from any cause } \\
\hline Alive or Censored & $34(67 \%)$ & $12(46 \%)$ & $22(88 \%)$ & $5(56 \%)$ & $29(69 \%)$ \\
\hline Dead & $17(33 \%)$ & $14(54 \%)$ & $3(12 \%)$ & $4(44 \%)$ & $13(31 \%)$ \\
\hline \multicolumn{6}{|l|}{ Cause of death } \\
\hline Alive or Censored & $34(67 \%)$ & $12(46 \%)$ & $22(88 \%)$ & $5(56 \%)$ & $29(69 \%)$ \\
\hline Death from oral cancer & $10(20 \%)$ & $8(31 \%)$ & $2(8.0 \%)$ & $2(22 \%)$ & $8(19 \%)$ \\
\hline Death from other causes & $5(9.8 \%)$ & $4(15 \%)$ & $1(4.0 \%)$ & $1(11 \%)$ & $4(9.5 \%)$ \\
\hline Unknown cause of death & $2(3.9 \%)$ & $2(7.7 \%)$ & $0(0 \%)$ & $1(11 \%)$ & $1(2.4 \%)$ \\
\hline \multicolumn{6}{|l|}{ Local recurrence } \\
\hline Censored/no event & $39(76 \%)$ & $17(65 \%)$ & $22(88 \%)$ & $7(78 \%)$ & $32(76 \%)$ \\
\hline Local recurrence & $12(24 \%)$ & $9(35 \%)$ & $3(12 \%)$ & $2(22 \%)$ & $10(24 \%)$ \\
\hline \multicolumn{6}{|l|}{ Regional recurrence } \\
\hline Censored/no event & $43(84 \%)$ & $19(73 \%)$ & $24(96 \%)$ & $8(89 \%)$ & $35(83 \%)$ \\
\hline Regional recurrence & $8(16 \%)$ & $7(27 \%)$ & $1(4.0 \%)$ & $1(11 \%)$ & $7(17 \%)$ \\
\hline \multicolumn{6}{|l|}{ Locoregional recurrence } \\
\hline Censored/no event & $38(75 \%)$ & $16(62 \%)$ & $22(88 \%)$ & $6(67 \%)$ & $32(76 \%)$ \\
\hline Locoregional recurrence & $13(25 \%)$ & $10(38 \%)$ & $3(12 \%)$ & $3(33 \%)$ & $10(24 \%)$ \\
\hline \multicolumn{6}{|l|}{ Distant metastasis } \\
\hline Censored/no event & $45(88 \%)$ & $21(81 \%)$ & $24(96 \%)$ & $9(100 \%)$ & $36(86 \%)$ \\
\hline Metastasis & $6(12 \%)$ & $5(19 \%)$ & $1(4.0 \%)$ & $0(0 \%)$ & $6(14 \%)$ \\
\hline
\end{tabular}

SOHND: Supraomohyoidal neck dissection; MRND: modified radical neck dissection; PORT: postoperative radiotherapy. ${ }^{1} \mathrm{n}(\%)$.

Table III. Estimated survival and probability of death from oral cancer or disease recurrence of recurrence by postoperative therapy and neck dissection.

\begin{tabular}{|c|c|c|c|c|c|c|c|c|}
\hline & \multicolumn{2}{|c|}{ Overall survival } & \multicolumn{2}{|c|}{ Death form oral cancer } & \multicolumn{2}{|c|}{ Regional recurrence } & \multicolumn{2}{|c|}{ Locoregional recurrence } \\
\hline & At 2 years & At 5 years & At 2 years & At 5 years & At 2 years & At 5 years & At 2 years & At 5 years \\
\hline Overall & $\begin{array}{c}74 \% \\
(63 \%-87 \%)\end{array}$ & $\begin{array}{c}64 \% \\
(51 \%-80 \%)\end{array}$ & $\begin{array}{c}20 \% \\
(11 \%-35 \%)\end{array}$ & $\begin{array}{c}22 \% \\
(13 \%-39 \%)\end{array}$ & $\begin{array}{c}18 \% \\
(9.3 \%-33 \%)\end{array}$ & $\begin{array}{c}18 \% \\
(9.3 \%-33 \%)\end{array}$ & $\begin{array}{c}25 \% \\
(15 \%-41 \%)\end{array}$ & $\begin{array}{c}28 \% \\
(17 \%-44 \%)\end{array}$ \\
\hline \multicolumn{9}{|l|}{ Treatment } \\
\hline Surgery only & $\begin{array}{c}57 \% \\
(40 \%-80 \%)\end{array}$ & $\begin{array}{c}41 \% \\
(25 \%-68 \%)\end{array}$ & $\begin{array}{c}30 \% \\
(16 \%-56 \%)\end{array}$ & $\begin{array}{c}36 \% \\
(20 \%-63 \%)\end{array}$ & $\begin{array}{c}33 \% \\
(18 \%-61 \%)\end{array}$ & $\begin{array}{c}33 \% \\
(18 \%-61 \%)\end{array}$ & $\begin{array}{c}42 \% \\
(26 \%-67 \%)\end{array}$ & $\begin{array}{c}42 \% \\
(26 \%-67 \%)\end{array}$ \\
\hline PORT & $\begin{array}{c}92 \% \\
(82 \%-100 \%)\end{array}$ & $\begin{array}{c}87 \% \\
(74 \%-100 \%)\end{array}$ & $\begin{array}{c}8.7 \% \\
(2.3 \%-33 \%)\end{array}$ & $\begin{array}{c}8.7 \% \\
(2.3 \%-33 \%)\end{array}$ & $\begin{array}{c}4.0 \% \\
(0.6 \%-27 \%)\end{array}$ & $\begin{array}{c}4.0 \% \\
(0.6 \%-27 \%)\end{array}$ & $\begin{array}{c}8.4 \% \\
(2.2 \%-32 \%)\end{array}$ & $\begin{array}{c}13 \% \\
(4.6 \%-38 \%)\end{array}$ \\
\hline \multicolumn{9}{|l|}{ Neck dissection } \\
\hline SOHND & $\begin{array}{c}78 \% \\
(55 \%-100 \%)\end{array}$ & $\begin{array}{c}52 \% \\
(27 \%-100 \%)\end{array}$ & $\begin{array}{c}25 \% \\
(7.5 \%-83 \%)\end{array}$ & $\begin{array}{c}25 \% \\
(7.5 \%-83 \%)\end{array}$ & $\begin{array}{c}14 \% \\
(2.3 \%-88 \%)\end{array}$ & $\begin{array}{c}14 \% \\
(2.3 \%-88 \%)\end{array}$ & $\begin{array}{c}38 \% \\
(15 \%-92 \%)\end{array}$ & $\begin{array}{c}38 \% \\
(15 \%-92 \%)\end{array}$ \\
\hline MRND & $\begin{array}{c}73 \% \\
(61 \%-88 \%)\end{array}$ & $\begin{array}{c}67 \% \\
(54 \%-84 \%)\end{array}$ & $\begin{array}{c}18 \% \\
(9.4 \%-36 \%)\end{array}$ & $\begin{array}{c}22 \% \\
(12 \%-41 \%)\end{array}$ & $\begin{array}{c}18 \% \\
(9.3 \%-36 \%)\end{array}$ & $\begin{array}{c}18 \% \\
(9.3 \%-36 \%)\end{array}$ & $\begin{array}{c}23 \% \\
(13 \%-40 \%)\end{array}$ & $\begin{array}{c}26 \% \\
(15 \%-44 \%)\end{array}$ \\
\hline
\end{tabular}

SOHND: Supraomohyoidal neck dissection; MRND: modified radical neck dissection; PORT: postoperative radiotherapy.

unanswered. Regional recurrence is not always caused by remnants of improperly treated regional disease, but can also be caused by a recurrent local disease. This requires a separate evaluation of isolated regional recurrences in the irradiated and non-irradiated groups as well. Due to the detailed follow-up records in the prospectively maintained study presented here, both RRFS and LRFS, could be investigated separately.
The recently published study on the benefit of adjuvant radiotherapy in oral cancer patients with a solitary nodal involvement was also based on retrospective records of the Taiwan Cancer Registry and the National Health Insurance (NHI) Research Database (11). Albeit meticulously performed in a high number of patients and considered adjusted hazard ratios, local and regional-free recurrences 


\section{A Overall survival}

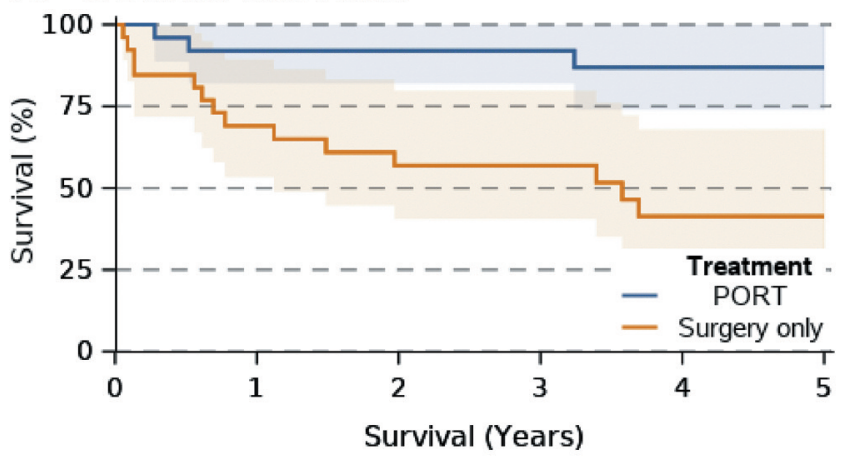

\section{B Death from oral cancer}

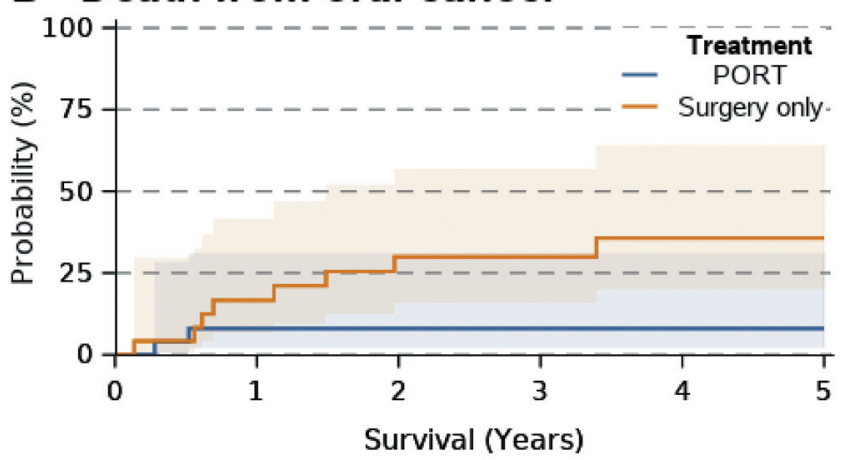

\section{Regional recurrence}

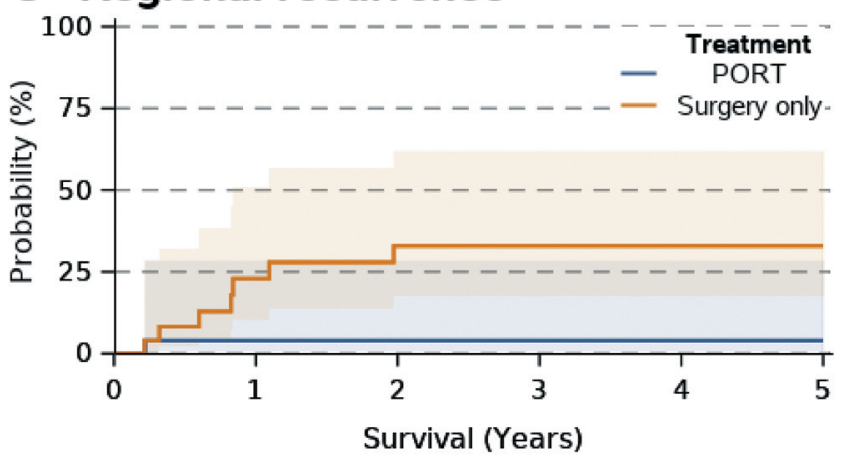

\section{Locoregional recurrence}

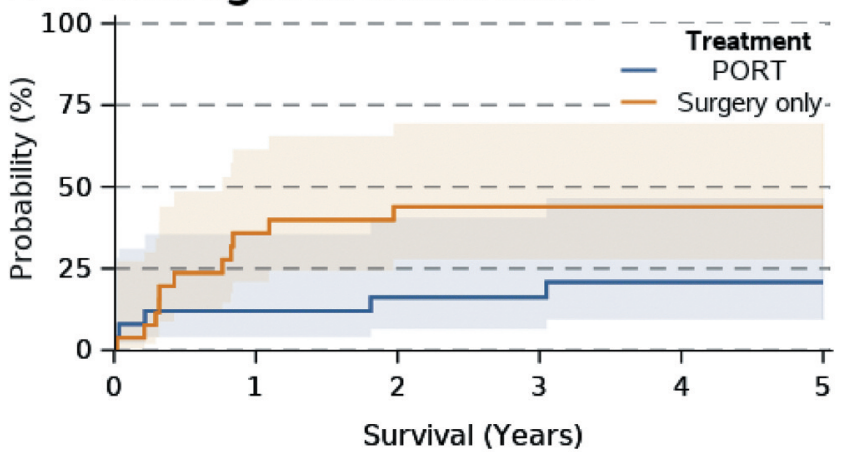

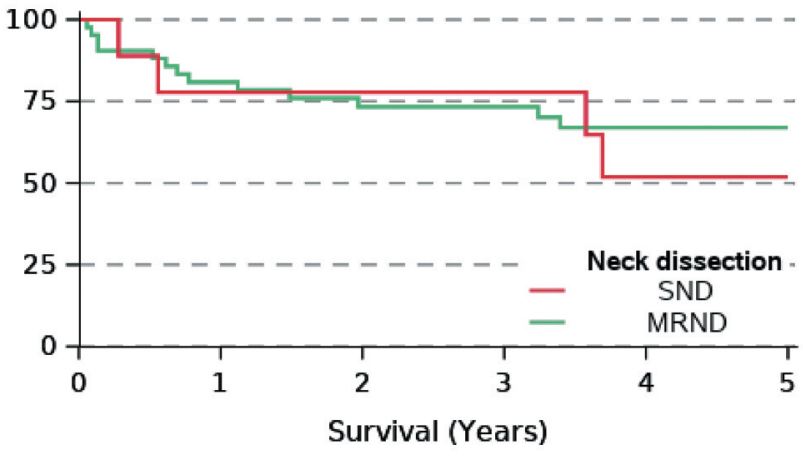
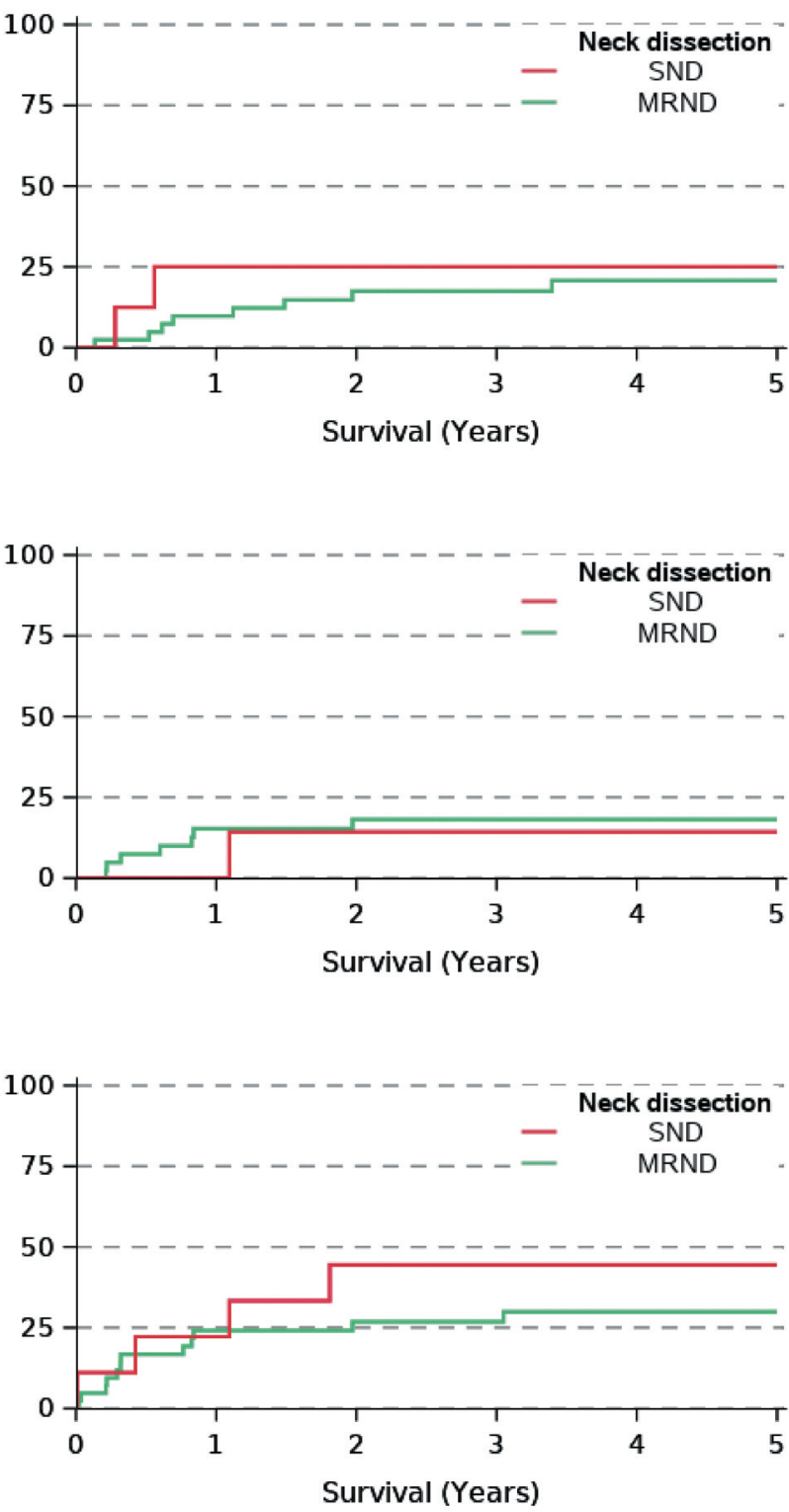

Figure 2. Kaplan-Meier and cumulative incidence function curves stratified by postoperative radiotherapy and by neck dissection. Yrs: Years; PORT: postoperative radiotherapy, SOHND: supraomohyoidal neck dissection; MRND: modified radical neck dissection. 
Table IV. Hazards ratios for different prognostic factors on overall survival, and disease-free survival. The models are stratified by tumor size and Charlson's comorbidity index score.

\begin{tabular}{|c|c|c|c|c|c|c|c|c|c|c|c|c|}
\hline & \multicolumn{3}{|c|}{ OS } & \multicolumn{3}{|c|}{ OCSS } & \multicolumn{3}{|c|}{ RRFS } & \multicolumn{3}{|c|}{ LRFS } \\
\hline & HR & $95 \% \mathrm{CI}$ & $p$-Value & HR & $95 \% \mathrm{CI}$ & $p$-Value & HR & $95 \% \mathrm{CI}$ & $p$-Value & HR & $95 \% \mathrm{CI}$ & $p$-Value \\
\hline Age & 1.05 & $0.99-1.11$ & 0.13 & & & & & & & & & \\
\hline \multicolumn{13}{|l|}{ Gender } \\
\hline Male & - & - & & - & - & & - & - & & - & - & \\
\hline Female & 0.81 & $0.25-2.59$ & 0.7 & & & & & & & & & \\
\hline \multicolumn{13}{|l|}{ Smoking } \\
\hline Never & - & - & & - & - & & - & - & & - & - & \\
\hline Former or current & 2.71 & $0.35-21.1$ & 0.3 & 1.59 & $0.15-16.5$ & 0.7 & 1.00 & $0.08-12.0$ & $>0.9$ & 0.50 & $0.07-3.78$ & 0.5 \\
\hline \multicolumn{13}{|l|}{ Alcohol consumption } \\
\hline None or moderate & - & - & & - & - & & - & - & & - & - & \\
\hline Excessive & 2.10 & $0.63-6.98$ & 0.2 & 0.88 & $0.21-3.60$ & 0.9 & 1.15 & $0.22-6.05$ & 0.9 & 1.95 & $0.46-8.35$ & 0.4 \\
\hline \multicolumn{13}{|l|}{ Treatment } \\
\hline Surgery only & - & - & & - & - & & - & - & & - & - & \\
\hline PORT & 0.22 & $0.05-1.03$ & 0.054 & 0.19 & $0.04-0.82$ & 0.026 & 0.07 & $0.01-0.69$ & 0.023 & 0.06 & $0.01-0.31$ & $<0.001$ \\
\hline \multicolumn{13}{|l|}{ Neck dissection } \\
\hline SOHND & - & - & & - & - & & - & - & & - & - & \\
\hline MRND & 0.83 & $0.25-2.76$ & 0.8 & 0.70 & $0.14-3.49$ & 0.7 & 1.30 & $0.15-11.1$ & 0.8 & 0.53 & $0.13-2.19$ & 0.4 \\
\hline
\end{tabular}

OS: Overall survival; OCSS: oral cancer specific survival; RRFS: regional recurrence-free survival; LRFS: locoregional recurrence-free survival; HR: hazard ratio; CI: confidence interval, SOHND: supraomohyoidal neck dissection; MRND: modified radical neck dissection; PORT: postoperative radiotherapy; Significant $p$-Values are shown in bold.

were not encountered separately. Nevertheless, the study elucidated an interesting clinical feature of tumors with higher potency for metastasis and thus explaining the benefit of PORT. Interestingly, only patients with pT2 pN1 tumors that were not located in the tounge had significantly better OS and LRFS if they were irradiated. Resection margin, depth of tumor invasion, perineural invasion, HPV-infection and whether it crossed the midline or not, were not considered in the analysis.

The main strength of the present study is the prospectively maintained data collection at the baseline and applying standard follow-up protocols. Although the patients' number is relatively low, many precisely obtained data within the follow-up prevent systematic bias. The fact that extending/completing of neck dissection to include all relevant cervical levels (I-V) does not influence neither the OS nor the RRFS, supports the hypothesis that a secondary removal of further uninvolved lymph node stations after assessment of the $\mathrm{pN} 1$ status does not improve the treatment outcome and is unnecessary.

In summary, the $\mathrm{pT} 1-2 \mathrm{pN} 1$ classification is a rare condition in patients with oral cancer, aggravating systematic investigation by regular randomized trials. The current understanding of treatment strategy is based on expert opinion and lacks scientific evidence. Among the retrospective studies, the current investigation presented here contributes to a better understanding of the beneficial effect of PORT on the OS, LRFS, and RRFS in patients with early stage $\mathrm{pN} 1$ tumors.

\section{Conflicts of Interest}

The Authors have no conflicts of interest to declare in relation to this study.

\section{Authors' Contributions}

UA and SH conceptualized the study and developed the methods. UA, DR, LT, DS and MF acquired histologic and follow-up data. UA conducted the statistical analyses. UA and SH interpreted the results and drafted the main manuscript. All Authors revised the final manuscript. SH supervised the study.

\section{References}

1 Moergel M, Meurer P, Ingel K, Wendt TG and Al-Nawas B: Effectiveness of postoperative radiotherapy in patients with small oral and oropharyngeal squamous cell carcinoma and concomitant ipsilateral singular cervical lymph node metastasis (pN1): A meta-analysis. Strahlenther Onkol 187(6): 337-343, 2011. PMID: 21603991. DOI: 10.1007/s00066-011-2206-x

2 Koyfman SA, Ismaila N, Crook D, D'Cruz A, Rodriguez CP, Sher DJ, Silbermins D, Sturgis EM, Tsue TT, Weiss J, Yom SS and Holsinger FC: Management of the neck in squamous cell carcinoma of the oral cavity and oropharynx: ASCO clinical practice guideline. J Clin Oncol 37(20): 1753-1774, 2019. PMID: 30811281. DOI: 10.1200/JCO.18.01921

3 German Guideline Program in Oncology (German Cancer Society, German Cancer Aid, AWMF): Evidence-based Guideline for Oral Cavity Cancer, Long version 3.0, Januar 2021, AWMF Registration Number: 007/100OL,https://www.leitlinienprogrammonkologie. 
de/leitlinien/mundhoehlenkarzinom/ [Last accessed on November 9, 2021]

4 Wolff $\mathrm{K}$, Rau A, Weitz J, Langer $\mathrm{T}$ and Zidane $\mathrm{M}$ : Aktualisierung der S3-Leitlinie "Diagnostik und Therapie des Mundhöhlenkarzinoms": Was ist neu? Der MKG-Chirurg 14(3): 177-183, 2021. DOI: 10.1007/s12285-021-00317-2

5 Quan H, Li B, Couris CM, Fushimi K, Graham P, Hider P, Januel JM and Sundararajan V: Updating and validating the Charlson comorbidity index and score for risk adjustment in hospital discharge abstracts using data from 6 countries. Am J Epidemiol 173(6): 676-682, 2011. PMID: 21330339. DOI: 10.1093/aje/kwq433

6 Kalbfleisch JD and Prentice RL: The statistical analysis of failure time data. John Wiley \& Sons, 2011.

7 Grambsch P and Therneau T: Proportional hazards tests and diagnostics based on weighted residuals. Biometrika 81(3): 515526, 2017. DOI: 10.1093/biomet/81.3.515

8 Weiss BG, Anczykowski MZ, Flach S, Spiegel JL, Kitz J, Bertlich M, Canis M, Jakob $M$ and Ihler F: Benefit of postoperative radiotherapy for early tumors with single ipsilateral lymph node metastasis. Laryngoscope 130(10): E530E538, 2020. PMID: 31837151. DOI: 10.1002/lary.28394

9 Liu T, David M, Batstone M, Clark J, Low TH, Goldstein D, Hope A, Hosni A and Chua B: The utility of postoperative radiotherapy in intermediate-risk oral squamous cell carcinoma. Int J Oral Maxillofac Surg 50(2): 143-150, 2021. PMID: 32616305. DOI: 10.1016/j.ijom.2020.06.006

10 Hasegawa T, Yanamoto S, Otsuru M, Kakei Y, Okura M, Yamakawa N, Yamada SI, Ota Y, Umeda M, Kirita T, Kurita H, Ueda M, Komori T and Japan Oral Oncology Group (JOOG): Multi-center retrospective study of the prognosis and treatment outcomes of Japanese oral squamous cell carcinoma patients with single lymph node metastasis and extra nodal extension. J Surg Oncol 117(8): 1736-1743, 2018. PMID: 29714825. DOI: $10.1002 /$ jso. 25083

11 Tsai CJ, Kuo YH, Wu HC, Ho CH, Chen YC and Yang CC: Adjuvant radiotherapy significantly increases neck control and survival in early oral cancer patients with solitary nodal involvement: a national cancer registry database analysis. Cancers (Basel) 13(15): 3742, 2021. PMID: 34359643. DOI: $10.3390 /$ cancers 13153742
12 Yıldırım T, Ozmen OA, Erişen L, Kasapoğlu F, Coşkun H, Basut $\mathrm{O}$, Onart S and Hizalan I: The role of selective neck dissection in pathological N1 squamous cell carcinomas of the head and neck. Kulak Burun Bogaz Ihtis Derg 21(2): 63-69, 2011. PMID: 21417967. DOI: 10.5606/kbbihtisas.2011.001

13 McMahon JD, Pitts R, Isbister J, Aslam-Pervez B, James A, McLellan D, Wright S, Wales CJ, McCaul J, Thomson E, Ansell MJ, Hislop WS, MacIver C, Devine JC and Carson E: Postoperative risk stratification in oral squamous cell carcinoma. Br J Oral Maxillofac Surg 58(4): 462-468, 2020. PMID: 32222310. DOI: 10.1016/j.bjoms.2020.02.026

14 Jiang W, Fang Z, Li Y, Wei H, Yang A, Chen Y and Zhang Q: Survival effect of postoperative radiotherapy combined with lymph nodes examined on cavity squamous cell carcinoma patients with stage T1-2N1M0: a propensity-adjusted SEER database analysis. 2021. DOI: 10.21203/rs.3.rs-386974/v1

15 Chen MM, Harris JP, Hara W, Sirjani D and Divi V: Association of postoperative radiotherapy with survival in patients with N1 oral cavity and oropharyngeal squamous cell carcinoma. JAMA Otolaryngol Head Neck Surg 142(12): 1224-1230, 2016. PMID: 27832255. DOI: 10.1001/jamaoto.2016.3519

16 Suresh K and Cramer JD: Postoperative radiation therapy vs observation for $\mathrm{pN} 1$ oral cavity squamous cell carcinoma. Head Neck 41(12): 4136-4142, 2019. PMID: 31589006. DOI: 10.1002/hed.25958

17 Xiang M, Holsinger FC, Gensheimer MF, Divi V, Pollom EL, Colevas AD, Le QT and Beadle BM: Postoperative observation versus radiotherapy for pathologic $\mathrm{N} 1$ oral cavity squamous cell carcinoma. Am J Clin Oncol 44(3): 99-104, 2021. PMID: 33417322. DOI: 10.1097/COC.0000000000000792

18 Shrime MG, Gullane PJ, Dawson L, Kim J, Gilbert RW, Irish JC, Brown DH and Goldstein DP: The impact of adjuvant radiotherapy on survival in T1-2N1 squamous cell carcinoma of the oral cavity. Arch Otolaryngol Head Neck Surg 136(3): $225-$ 228, 2010. PMID: 20231637. DOI: 10.1001/archoto.2010.22

Received October 5, 2021

Revised November 7, 2021

Accepted November 8, 2021 